\section{Challenges in Prenatal Diagnosis of Cornelia de Lange Syndrome: A Case Study}

\section{Abstract}

In this case study we describe the prenatal and postnatal course of a patient with Cornelia de Lange Syndrome (CdLS) focusing on the prenatal diagnostic challenges. During a routine prenatal ultrasonographic survey, a 19 weeks female fetus was found to have several anatomical anomalies including poorly visualized cavum septum pellucidum. Fetal growth was normal at that time. Fetal brain MRI at 22 weeks demonstrated an enlarged extra-axial space and scalp edema, but no other apparent anomalies. Amniocentesis revealed normal karyotype and chromosomal microarray. Postnatally the infant possessed physical features suggestive of CdLS (growth restriction, microcephaly, limb deformities), later genetically confirmed with a mutation in NIPBL gene. Postnatal brain MRI demonstrated brachycephaly, immature frontal lobe sulcation, and partial agenesis of the corpus callosum that were not seen on the fetal MRI. The case supports previous radiological findings, emphasizing the importance of subtle ultrasonographic and MRI features that potentially could lead to earlier detection of the syndrome.

Keywords: Cornelia De Lange Syndrome; Diagnostic Imaging; Prenatal Ultrasonography; Magnetic Resonance Imaging

Received: May 08, 2020; Accepted: June 04, 2020; Published: June 11, 2020

\section{Michael Stanley ${ }^{1}$, Tomo Tarui ${ }^{2}$ and Alexa Craig $^{3 *}$}

1 Tufts University School of Medicine, 145 Harrison Avenue, Boston, MA 02111, USA

2 Mother Infant Research Institute, Tufts Medical Center, 800 Washington Street, Boston, MA 02111, USA

3 Department of Pediatrics, Maine Medical Center, 55 Spring St, Scarborough, ME, 04074, USA

\section{*Corresponding author: Alexa Craig \\ craiga@mmc.org \\ Department of Pediatrics, Maine Medical Center, 55 Spring St, Scarborough, ME, 04074, USA.}

Citation: Stanley M, Tarui T, Craig A (2020) Challenges in Prenatal Diagnosis of Cornelia de Lange Syndrome: A Case Study. J Rare Disord Diagn Ther Vol.6 No.3:3

\section{Introduction}

Cornelia de Lange Syndrome (CdLS) is a usually sporadic disorder, although cases of familial X-linked dominant, autosomal dominant, and paternal gonadal mosaicism modes of inheritance have been reported. Seven genes including NIPBL (Nipped-BLike Protein), SMC1A (Structural Maintenance Of Chromosomes 1A), SMC3 (Structural Maintenance of Chromosomes 3), RAD21 (Human Homolog of Schizosaccharomyces pombe radiation sensitive mutant 21), BRD4 (Bromodomain containing 4), HDAC8 (histone deacetylase 8) and ANKRD11 (Ankyrin repeat domain 11) have been associated with CdLS. The prevalence of CdLS is roughly $0.5-10$ per 100,000 births [1], and this syndrome is typically diagnosed at birth by characteristic facial features (well defined, arched eyebrows with synophrys, long philtrum, thin lips, and crescent-shaped mouth), microbrachycephaly and limb defects. Affected individuals may develop severe intellectual disability, behavioral problems (such as self-injurious behavior), autism, and proportionately small stature [2,3]. One of the largest and most recent reviews of prenatal CdLS (53 cases) demonstrated that fetal growth restriction (FGR) appeared in $81 \%$ and was detectable as early as 16 weeks. The characteristic facial features were detected in $49 \%$ of cases at $19-20$ weeks [4].
Only one publication has thus far documented fetal brain MRI findings in an affected fetus [5]. This 22 week fetus had frontal bossing, subcutaneous edema, and small cerebral hemispheres. The current case is presented to highlight the similarity between the published fetal MRI brain imaging in our case and to suggest that fetal MRI may be beneficial in the evaluation of suspected CdLS cases. Early identification of this syndrome may be beneficial for counselling the parents of affected fetuses.

\section{Case Report}

During a routine fetal anatomic survey at $196 / 7$ weeks gestation, a female fetus was found to have a single umbilical artery, absent nasal bone, echogenic kidneys, scalp edema, and a poorly visualized cavum septum pellucidum. Fetal growth was normal at that time (Table 1). For further characterization of the septum pellucidum abnormality, fetal brain MRI performed at $226 / 7$ weeks gestation (Figure 1) noted a remarkably enlarged extra-axial space and scalp edema (solid arrows) but was thought otherwise to be structurally normal. Amniocentesis was performed, and both limited karyotype and microarray were normal. With no definitive diagnosis, the parents decided to continue the 
Table 1: Fetal growth parameters at 19 6/7 weeks and 34 2/7 weeks. The later development of fetal growth restriction, a cardinal feature of CdLS, delayed detection of the syndrome.

\begin{tabular}{|c|c|c|c|c|c|c|}
\hline \multirow{2}{*}{ Variables } & \multicolumn{3}{|c|}{$196 / 7$ Weeks } & \multicolumn{3}{|c|}{34 2/7 Weeks } \\
\hline & Measures & Dates & Range & Measure & Dates & Range \\
\hline BPD & $44.8 \mathrm{~mm}$ & $19 w 4 d$ & $19 w 0 d-20 w 1 d$ & $81.1 \mathrm{~mm}$ & $32 \mathrm{w} 4 \mathrm{~d}$ & $31 w 4 d-33 w 4 d$ \\
\hline $\mathrm{HC}$ & $157.3 \mathrm{~mm}$ & $18 \mathrm{w} 4 \mathrm{~d}$ & $17 w 1 d-20 w 1 d$ & $276.2 \mathrm{~mm}$ & $30 \mathrm{w} 1 \mathrm{~d}$ & $27 w 1 d-33 w 1 d$ \\
\hline AC & $137.3 \mathrm{~mm}$ & $19 w 1 d$ & $18 w 3 d-19 w 6 d$ & $265.7 \mathrm{~mm}$ & $30 w 5 d$ & $29 w 5 d-31 w 5 d$ \\
\hline FL & $28.8 \mathrm{~mm}$ & $18 w 6 d$ & $17 w 0 d-20 w 5 d$ & $58.6 \mathrm{~mm}$ & $30 w 4 d$ & $27 w 5 d-33 w 4 d$ \\
\hline EFW & $274 \mathrm{~g}$ & & & $1687 \mathrm{~g}\left(7^{\text {th }} \%\right)$ & & \\
\hline
\end{tabular}

BPD: Biparietal Diameter; HC: Head Circumference; AC: Abdominal Circumference; FL: Femur Length; EFW: Estimated Fetal Weight

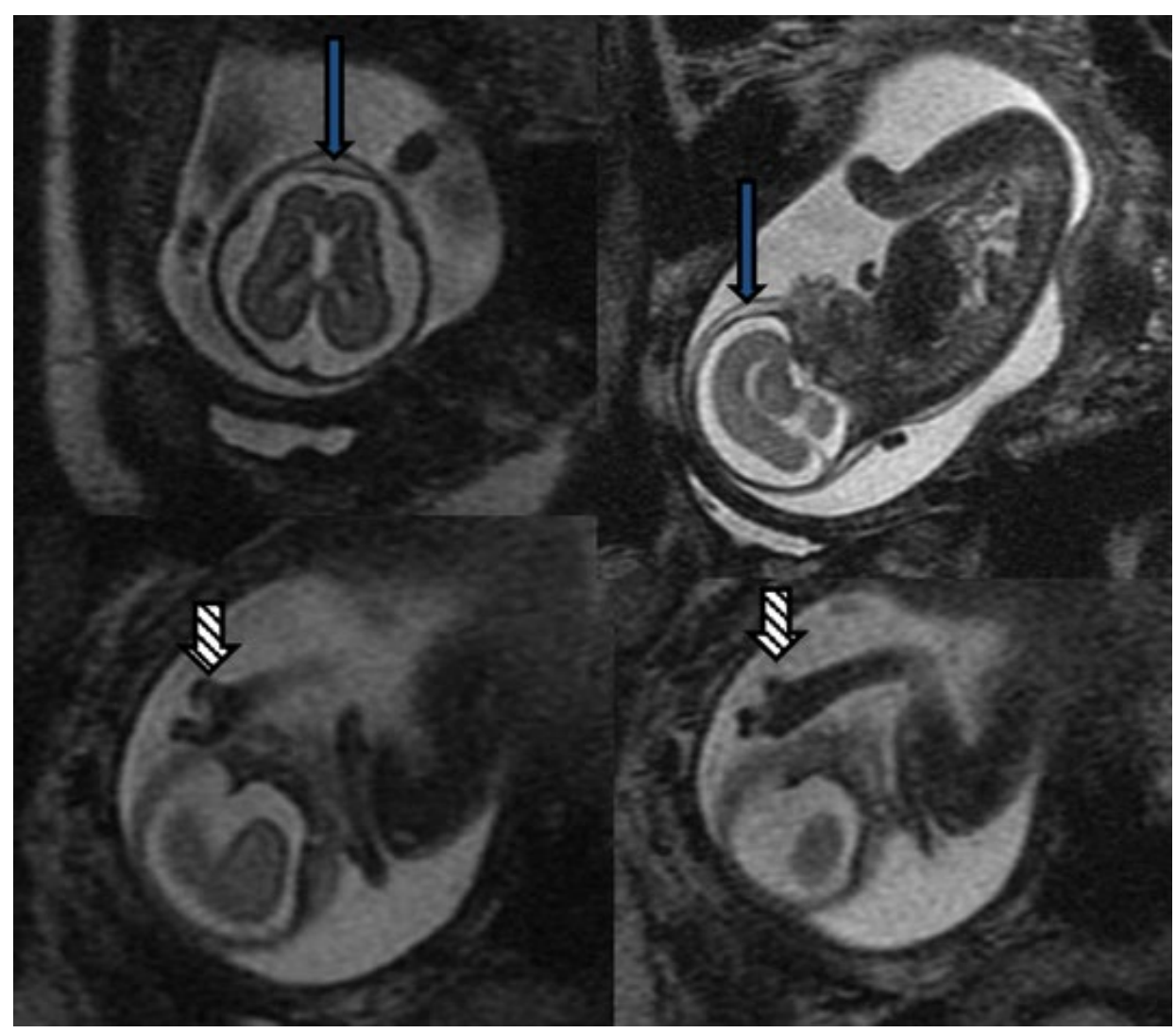

Figure 1 Fetal MRI Findings: Top left and right-T2 weighted fetal MRI images revealing enlarged extra-axial space for gestational age of $226 / 7$ weeks and subscalp edema indicated by blue arrows. Bottom left and right-T2 weighted fetal MRI images revealing hand deformity (striped arrow).

pregnancy. The patient was born prematurely at 36 weeks and demonstrated clinical findings consistent with CdLS, namely late onset FGR, microcephaly (head circumference $29 \mathrm{~cm}$ ), copious dark hair present over the scalp with low anterior and very low posterior hairline, and highly arched eyebrows. The fourth digit on the left hand was missing. The right thumb was hypoplastic, and the right fifth digit had striking distal phalange hypoplasia. She had bilateral optic nerve colobomas. Using Cornelina de Lange
Syndrome NextGen Sequencing panel (Prevention Genetics, WI, USA), she was confirmed to be heterozygous for c.2653dup G resulting in premature termination of the NIPBL gene. Neonatal brain MRI at 36 5/7 weeks was notable for microbrachycephaly, a simplified gyral pattern of frontal lobes, and partial agenesis of the corpus callosum (Figure 2). She was discharged from the neonatal intensive care on day of life 49 with a gastrostomy tube in place. 


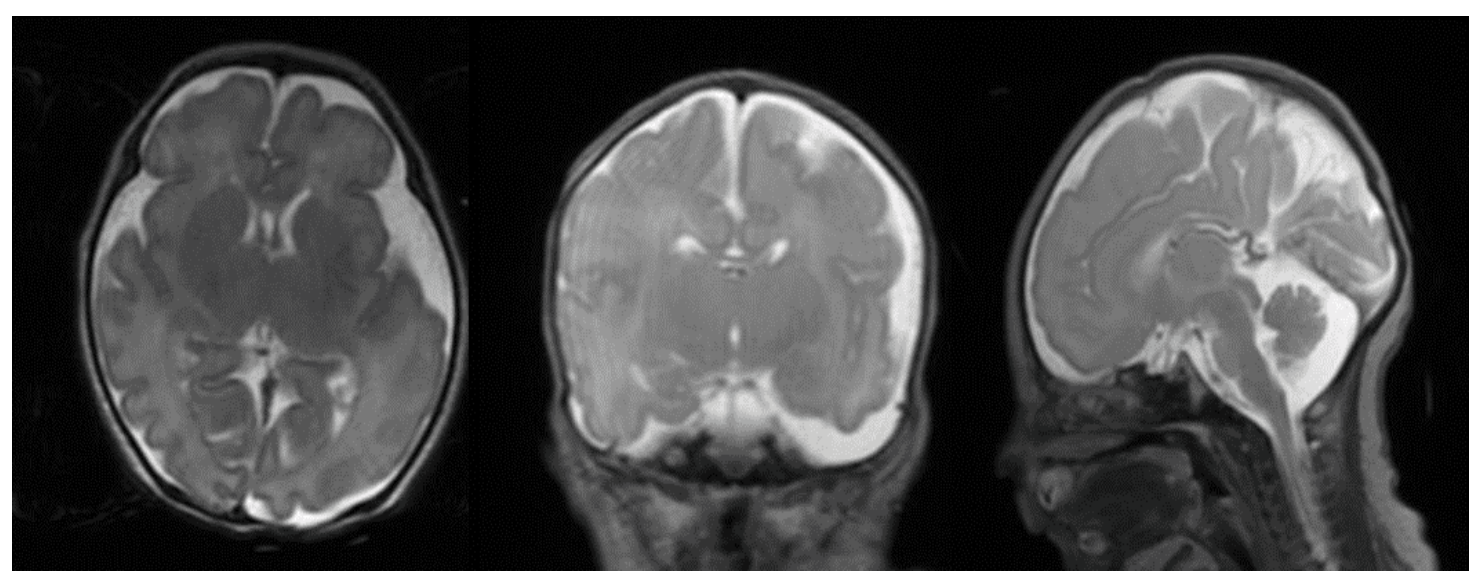

Figure 2 Neonatal MRI findings: T2 weighted neonatal MRI images (axial, coronal and sagittal planes) revealing microbrachycephaly, simplified frontal gyral folding pattern and callosal hypoplasia at gestational age 36 5/7 weeks.

\section{Discussion}

Prenatal diagnosis ofCdLSiscomplicated given the wide phenotypic variability in presentation. Large defects such as diaphragmatic hernias, obvious cardiac defects, or severe limb reduction defects can provide significant diagnostic and prognostic clues for fetuses affected by CdLS. These more obvious defects were not present in our case, a fact that contributed to the difficulty with early recognition, however given the rarity of this disorder, even when limb reduction defects are present, the diagnosis is not always made prior to birth [6]. In our case, as with many other postnatally diagnosed cases, the milder phenotype did not prompt consideration of a CdLS in the prenatal diagnosis, due to the absence of its cardinal features such as FGR, microcephaly and limb deformities. Our case eventually developed FGR and microcephaly, although it was not appreciated until the third trimester of pregnancy (estimated fetal weight was at $19^{\text {th }}$ percentile at 28 weeks, $10^{\text {th }}$ percentile at 32 weeks and then $7^{\text {th }}$ percentile at 34 weeks), which further contributed to difficulty with diagnosis. Many affected infants develop FGR between 20-25 weeks. However, this is certainly a non-specific finding, and other abnormalities (i.e., facial or limb) would need to be carefully investigated for specific diagnosis. The abnormal facial features associated with CdLS are subtle on ultrasound and MRI, and although in retrospect the hand deformity was apparent on MRI (Figure 1 striped arrows), it was not appreciated prior to the birth. Routine screening studies for genetic abnormalities of the fetus including the karyotype and microarray that were performed following amniocentesis in this pregnancy do not have the sensitivity to diagnose disorders such as CdLS. This highlights the importance of recognizing the phenotypic features to permit targeted genetic testing with a gene sequencing panel specific to CdLS that could be performed during the pregnancy. This case illustrates the importance of increasing awareness of the milder phenotypic presentations of CdLS and encouraging the use of fetal MRI to characterize subtler abnormalities affecting both brain and limbs. One prior report has characterized a CdLS fetus on fetal MRI with scalp edema and frontal bossing. While frontal bossing was not appreciated on our case, the appearance of scalp edema is remarkably similar [5]. Other authors have described postnatal neuroimaging findings including cerebellar vermian hypoplasia, cerebellar vermian agenesis, ventriculomegaly, dangling choroid plexus, and agenesis of corpus callosum [4] in affected infants. Additionally, an MRI of a 15-year old male with CdLS showed mild enlargement of the $3^{\text {rd }}$ and $4^{\text {th }}$ ventricles, but sparing both cortex and putamen of any structural lesions [7].

\section{Conclusion}

In prenatal cases, limb abnormalities are one of the more helpful findings leading to an antenatal diagnosis with 14/15 antenatal diagnosed fetus having limb anomalies in one case series. Had the limb abnormality in our case been appreciated on either ultrasound or fetal MRI, it is conceivable that an antenatal diagnosis could have been made. We suggest that consideration be given to repeating fetal MRI to aid in diagnosis and not limiting fetal MRI to the evaluation of the cranium, a factor which might have permitted the limb reduction defects to be appreciated in this case. Given the severity of outcomes of affected CdLS children and the lack of a good correlation between milder phenotype and developmental outcome, parents need early diagnosis for optimal prenatal counseling. 


\section{References}

1 Boyle MI, Jespersgaard C, Brondum-Nielsen K, Bisgaard AM, Tumer Z, et al. (2015) Cornelia de Lange syndrome. Clin Genet 88: 1-12.

2 Barisic I, Tokic V, Loane M, Bianchi F, Calzolari E, et al. (2008) Descriptive epidemiology of Cornelia de Lange syndrome in Europe. Am J Med Genet A 146A: 51-59.

3 Kline AD, Krantz ID, Sommer A, Kliewer M, Jackson LG, et al. (2007) Cornelia de Lange syndrome: A clinical review, diagnostic and scoring systems, and anticipatory guidance. Am J Med Genet A 143A: 12871296.

4 Clark DM, Sherer I, Deardorff MA, Byrne JLB, Loomes KM, et al. (2012) Identification of a prenatal profile of Cornelia de Lange syndrome (CdLS): a review of 53 CdLS pregnancies. Am J Med Genet A 158A: 1848-1856.

5 Lalatta F, Russo S, Gentilin B, Spaccini L, Boschetto C, et al. (2007) Prenatal/neonatal pathology in two cases of Cornelia de Lange syndrome harboring novel mutations of NIPBL. Genet Med 9: 188-194.

6 Kinjo T, Mekaru K, Nakada M, Nitta H, Masamoto H, et al. (2019) A Case of Cornelia de Lange Syndrome: Difficulty in Prenatal Diagnosis. Case Rep Obstet Gynecol 2019: 4530491.

7 Silva-Hernandez F, Rodriguez-Cuadrado GI, Martin-Ruaigip RJ, Barreras-Ávila L, González-Chevere B, et al. (2015) Functional brain imaging in Cornelia de Lange Syndrome: A case report and literature review. Bol Asoc Med PR 107: 73-77. 